\title{
La afectación del derecho al trabajo de los servidores públicos ocasionado por los contratos ocasionales en el Ecuador
}

URL: http://revistas.uta.edu.ec/erevista/index.php/bcoyu/article/view/720 Dol: http://dx.doi.org/10.31164/bcoyu.22.2019.720

\author{
Bryan Velaña-Bayas ${ }^{1}$ \\ Fecha de recepción: 21 de marzo de 2019 \\ Fecha de aceptación: 20 de junio de 2019
}

\section{Resumen}

El Estado Ecuatoriano reconoce el derecho al trabajo de los servidores públicos en todas sus formas; tanto la Constitución del Ecuador como la Ley Orgánica del Servicio Público. El presente estudio analiza la normativa antes mencionada y resoluciones judiciales; y se determina que el ordenamiento jurídico es ineficiente lo cual vulnera derechos y principios constitucionales. La metodología que se utilizó fue crítico propositivo con enfoque cuali-cuantitativo, mediante la utilización de revistas, libros, jurisprudencia, leyes, los mismos que permitieron dar sustento legal.

Palabras clave: Derecho, trabajo, servidores públicos, contratos ocasionales.

\section{Abstract}

The State of Ecuador recognizes the right to work of public servants in all its forms both the Constitution of Ecuador and the organic law of the public service. The present study analyzes the aforementioned regulations and judgments; and determines that the legal system is inefficient, which violates rights and constitutional principles. The methodology used was proactive critical qualitative-quantitative, approach the use of books, journals, law, laws, which allowed legal support.

Keywords: Right, labour, public servants, casual contracts.

\section{Introducción}

La presente investigación se realizó debido a que las entidades del Estado efectúan contratos ocasionales a los servidores, existiendo la posibilidad de dejar sin el sustento de hogar para diferentes familias ecuatorianas, esto, por la modalidad de contratación, por el corto tiempo de la relación laboral existente. El estudio busca ayudar y facilitar a las entidades nominadoras y a los servidores públicos en el fortalecimiento y el fomento de los concursos de méritos y oposición propuesto por el Ministerio del Trabajo a nivel nacional. La importancia de este estudio radica en determinar si el Estado cumple lo establecido en el artículo 229 de la Constitución del Ecuador; para lo cual se pretende establecer criterios jurídicos para con ello otorgar todos los derechos y obligaciones que acoge el derecho antes señalado.

El trabajo contiene la conceptualización de temas como: derecho al trabajo, servidores públicos y también aborda los contratos ocasionales en el Ecuador. Respecto a los antecedentes investigativos del tema no se han realizado trabajos que guardan estrecha concordancia, entre las más relevantes se puede enunciar: Muñoz (2014) Salazar (2015), Abad (2016), Valencia (2016), Gavilánez (2018),

No se respeta el principio de igualdad ante la ley, reconocido en la Constitución del Ecuador (2008) en su artículo 66 numeral 4, puesto que ciertos funcionarios son contratados bajo la modalidad de contratos ocasionales dentro de la administración pública, restringiéndoles ciertos derechos (Muñoz, 2014).

El Estado no sanciona la suscripción de contratos ocasionales, por cuanto, se deben realizar concursos de méritos y oposición, el mismo que representa la cuarta parte de ellos, que a su vez son destituidos de sus puestos de manera directa e inmediata, ocasionando inseguridad e inestabilidad laboral que es producto de un sistema ineficaz (Salazar, 2015).

Los contratos ocasionales han generado inestabilidad en los funcionarios, un desgaste de recursos del Estado (capacitaciones) quienes tienen que posteriormente ser separados de la institución al término de su contrato (Abad, 2016). Sin embargo, la Ley Orgánica de Servicio Público no está vinculada con la Constitución del Ecuador (2008), porque solo garantiza los derechos de los servidores públicos que perdurarán o que estarán durante un tiempo prolongado o para siempre en su cargo (Valencia, 2016).

El Ecuador es un Estado Constitucional de derechos y justicia, no obstante, no proporciona estabilidad laboral a ciertos servidores públicos conforme los contratos ocasionales, teniendo la obligación jurídica o legal no cumple lo establecido en los artículos 1 y 332 de la Constitución del Ecuador (Gavilánez, 2018).

\section{Fundamentos Teóricos}

\section{Derecho al trabajo}

En el Ecuador, dentro de la Constitución del Ecuador (2008), se define que, "el trabajo es un derecho y un deber social, y un derecho económico, fuente de realización personal y base de la economía" (art. 33).

De lo citado, se interpreta que la legislación ecuatoriana reconoce y garantiza el derecho al trabajo en todas sus formas, con la finalidad de otorgar una vida digna a las personas estableciendo derechos y obligaciones conforme la ley.
En cuanto, la Carta Andina (2002), establece que el trabajo como una oportunidad parar gozar de una vida digna y satisfacer los intereses personales y familiares como ropa, comida y vivienda (art. 24).

Según Ostau de Lafont (2009) en su obra exteriorizó que, el derecho laboral es de carácter proteccionista, ya que dentro de la relación laboral el trabajador no tiene las mismas aptitudes para poder competir con el empleador, de esta manera ante cualquier clase de conflicto la parte perjudicada será el trabajador, por lo que el Estado tiene la obligación de precautelar el bienestar del más débil.

Mientras tanto, el derecho al trabajo es una rama jurídica que regula las relaciones del ser humano en el ámbito de sus labores; es decir la función como tal que desempeña en relación de dependencia, con consecuencias individuales y colectivas con el fin del progreso general del pueblo (Balbín, 2015).

Es decir, el trabajo es un derecho reconocido a nivel nacional como internacional el mismo que es fuente de realización personal y base de la economía para garantizar una vida digna en sociedad, para lograr mejorar su calidad de supervivencia.

\section{Servidores Públicos}

Está conformado por personal humano, el mismo que debe tener una capacidad operativa, administrativa para la ejecución correcta dentro de un cargo público para cumplir los intereses de la población; protegiendo los intereses institucionales.

Al tratar de definir al servidor público, según Parra (2005), "es una persona natural, que presta servicios personales a una entidad del Estado, con el propósito de recibir una remuneración (p.6).

Por tanto, en la Constitución de la República del Ecuador (2008): determina que, serán servidoras o servidores públicos todas las personas que en cualquier forma o a cualquier título trabajen, presten servicios o ejerzan un cargo, función o dignidad dentro del sector público" (art. 229).

Además, cabe mencionar que sus derechos son irrevocables, es decir que no puede desistir de ellos bajo ningún concepto.

De lo mencionado, se deduce que, los servidores públicos son personas humanas que ejercen derechos y contraen obligaciones a título personal contratadas por el Estado, las mismas que prestan sus servicios personales, lícitos bajo remuneración y tienen los mismos derechos que otros, por lo que tienen relación de dependencia y subordinación, es decir, que una entidad estatal pone horarios de trabajo a igual que todos los funcionarios y realizan sus actividades administrativas.

Los servidores públicos en el Ecuador, en su mayoría son contratados por las diferentes funciones del Estado: Función Ejecutiva, Función Legislativa, Función Judicial bajo servicios ocasionales, los cuales asciende a 95.294 según el año 2017, conforme se presenta en la tabla 1.

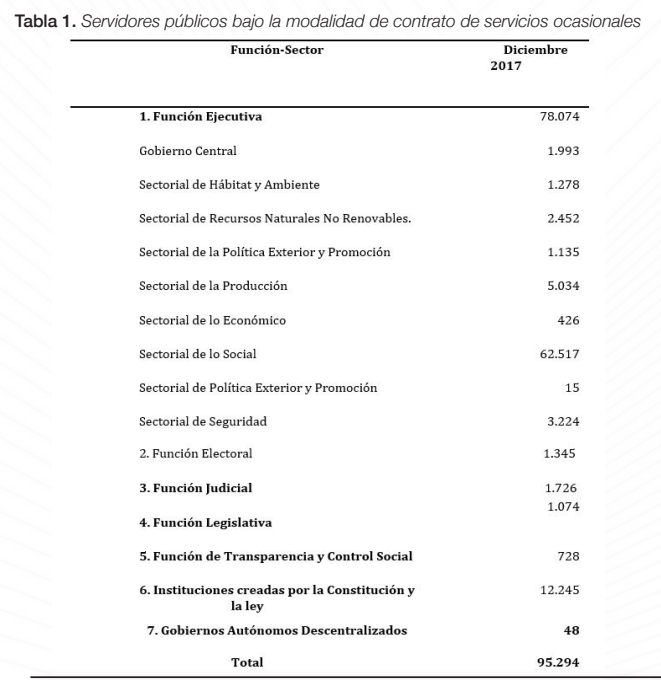

Fuente: Ministerio de Trabajo-Distributivo por el Ministerio de Finanzas. Corte al 05 de marzo de 2019 
Mientras tanto, a enero 2018, el número de servidores públicos que son contratados bajo la modalidad del contrato ocasional se redujo, según se muestra en la tabla 2.

\begin{tabular}{|c|c|}
\hline Función-Sector & Enero-2018 \\
\hline 1. Función Ejecutiva & 76.123 \\
\hline Gobierno Central & 1.443 \\
\hline Sectorial de Hábitat y Ambiente & 1.207 \\
\hline $\begin{array}{l}\text { Sectorial de infraestructura y de Recursos } \\
\text { Naturales No Renovables. }\end{array}$ & 2.265 \\
\hline Sectorial de la Política Exterior y Promoción & 1.091 \\
\hline Sectorial de la Producción & 4.501 \\
\hline Sectorial de lo Económico & 425 \\
\hline Sectorial de lo Social & 62.294 \\
\hline Sectorial de Política Exterior y Promoción & 14 \\
\hline Sectorial de Seguridad & 2.883 \\
\hline 2. Función Electoral & 1.830 \\
\hline 3. Función Judicial & 1.670 \\
\hline 4. Función Legislativa & 1.017 \\
\hline 5. Función de Transparencia y Control Social & 780 \\
\hline $\begin{array}{l}\text { 6. Instituciones creadas por la Constitución y } \\
\text { la ley }\end{array}$ & 10.872 \\
\hline 7. Gobiernos Autónomos Descentralizados & 49 \\
\hline Total & 92.341 \\
\hline
\end{tabular}

Fuente: Ministerio de Trabajo-Distributivo por el Ministerio de Finanzas. Corte al 05 de marzo de 2019.

De acuerdo al Ministerio de Trabajo-Distributivo por el Ministerio de Finanzas, las instituciones con más contratos de servicios son: Ministerio de Salud Pública con 28.772, Ministerio de Educación con 27.287, Senescyt con 2.883, Ministerio de Agricultura y Ganadería con 2.625, Universidad de Guayaquil con 1.910, Consejo Nacional Electoral con 1.817, Ministerio de Inclusión Económica y Social con 1.636, Ministerio de Justicia con 1.028, Asamblea Nacional con 1.017. En la tabla 3 se muestra la información en mención:

\begin{tabular}{lc}
\multicolumn{2}{c}{ Tabla 3. Instituciones con más contratos de servicios } \\
\hline Institución & Cantidad \\
\hline Ministerio de Salud Pública & 28.772 \\
Ministerio de Educación & 27.287 \\
Senescyt & 2.883 \\
Ministerio de Agricultura y Ganadería & 2.625 \\
Universidad de Guayaquil & 1.910 \\
Consejo Nacional Electoral & 1.817 \\
Ministerio de Inclusión Económica y Social & 1.636 \\
Ministerio de Justicia & 1.028 \\
Asamblea Nacional & 1.017 \\
& \\
Total & 68.975
\end{tabular}

Fuente: Ministerio de Trabajo-Distributivo por el Ministerio de Finanzas. Corte al 05 de marzo de 2019.

Derechos de los servidores y servidoras públicas en el Ecuador

Toda persona tiene derecho al trabajo al ser un deber social, por cuanto García (2012), determina que "es el conjunto de normas que protegen los esfuerzos materiales e intelectuales de la vida humana" (p. 17). Es decir, es una prestación de trabajo libre, contralada y remunerada por el empleador quien logra una ganancia económica.

Sobre sus derechos la Constitución del Ecuador menciona que los ciudadanos ecuatorianos gozaran de derechos establecidos en la normativa antes mencionada, en ese sentido, el Estado promueve el empleo, genera políticas, subempleo para garantizar un ambiente oportuno que permita al trabajador contar con seguridad, bienestar, favoreciendo cualquier cuestión laboral (Valencia, 2016).

Finalmente, uno de los derechos primordiales es la estabilidad laboral, misma que se encuentra establecida en el artículo 229 de la Constitución del Ecuador, por lo que es un derecho fundamental e irrenunciable. Es recomendable, regular y coordinar con las demás instituciones del Estado con el fin de evitar y reducir el alto índice de servidores públicos que son contratados bajo la forma de contratos ocasionales.
Causas de la Inestabilidad Laboral

Para la Psicología Organizacional, hace hincapié que es motivo de preocupación la situación que enfrentan los trabajadores dominado por criterios de efectividad y eficiencia, situación que genera percepción de inseguridad frente a la permanencia en el empleo, a posibilidades futuras y a la propia supervivencia, a su vez tiempo que se les exigen altos grados de compromiso con la organización (Peralta, Santofimio, \& Segura, 2007).

La estabilidad laboral es uno de los principios fundamentales que erigen a las relaciones de trabajo y descaderarle de otros derechos en las prácticas de la flexibilización con tonalidades sociales y amplia valoración de los derechos de los trabajadores (Montserrat, 2014).

Según Durán (2010), deja en firme que la Organización Mundial de Salud, considera al estrés en el trabajo como una de las causas que afecta la estabilidad emocional de los trabajadores y trabajadoras y esto gravemente daña el correcto desenvolvimiento de sus actividades en su lugar de trabajo.

\section{Contratos ocasionales en el Ecuador}

Esta reconocido en la Ley Orgánica del Servicio Público (LOSEP), el mismo que manifiesta que: La suscripción de contratos de servicios ocasionales seră autorizada de forma excepcional por la autoridad nominadora, para satisfacer necesidades institucionales no permanentes, previo el informe motivado de la Unidad de Administración del Talento Humano, siempre que exista la partida presupuestaria y disponibilidad de los recursos económicos para este fin (art. 58).

De lo citado, se deduce que la autoridad nominadora es aquella que formaliza los nombramientos de los servidores públicos en el Ecuador (Ministerio de Relaciones Laborales), a su vez se interpreta que debe existir el presupuesto económico con la finalidad de contratación para con ello otorgar los beneficios de ley, por lo que el Estado debe tener mecanismos eficientes y oportunos.

Estos contratos no otorgan licencias y tampoco nombramientos de carácter temporal. Por lo cual para estudios no se permiten estas licencias conforme el art. 58 de la LOSEP.

Según la Ley Orgánica del Servicio Público (2010): Si una institución no cuenta con personal suficiente, la misma podrä volver a contratar por única vez hasta por doce meses adicionales; así como en casos de personas con discapacidad debidamente calificadas por la Autoridad competente y el de las mujeres embarazadas y en estado de gestación. En este último caso, la vigencia del contrato durará hasta finalizar la etapa de lactancia (art. 58)

De lo mencionado, se puede mencionar que a los servidores públicos la normativa antes mencionada no proporciona todos los beneficios de ley, excluyendo a los trabajadores que son contratados bajo esta modalidad.

\section{Los contratos de trabajo en el Ecuador}

A lo largo de la historia se han creado diferentes normativas relacionadas al contrato de trabajo con la finalidad de cubrir las necesidades de la sociedad o comunidad industrial. El contrato individual de trabajo, la legislación en ese sentido establece que es: "el convenio en virtud del cual una persona se compromete para con otra u otras a prestar sus servicios lícitos y personales, bajo su dependencia, por una remuneración fijada por el convenio, la ley, el contrato colectivo o la costumbre" (Código de trabajo, 2005, art. 8).

Es el acuerdo que se da entre una persona o más personas para prestar un servicio o actividad personal, siempre que ésta sea lícita, y esta debe ser remunerada, esta remuneración no puede ser menor de lo establecido dentro de la misma ley, en la cual el trabajador estará bajo dependencia del empleador, además que las partes pueden pactar otras obligaciones o beneficios que la ley lo permita (Maldonado, 2017).

En cuanto a la clasificación el Código de Trabajo (2005), determina que puede ser: Expreso o tácito, y el primero, escrito o verbal; a sueldo, jornal, en participación y mixto; por tiempo indefinido, de temporada, eventual y ocasional; por obra cierta, por obra o servicio determinado dentro del giro del negocio, por tarea y a destajo; y, individual, de grupo o por equipo (art. 11)

\section{El contrato de trabajo en otras legislaciones}

En Colombia, la normativa respecto a la regulación de los contratos laborales se determina en la ley 789 del 2002, misma que expresa que es un límite temporal específico de manera clara en el contrato, mismo que puede ser prorrogado por cierto tiempo siempre y cuando exista la predisposición de las partes. En ese sentido, los contratos ocasionales en esa legislación son llamados temporales o accidentales predeterminado en el art. 6 del Código Sustantivo de trabajo, por lo que su duración es no mayor de un mes; es decir, se contrata los servicios cuando surge la necesidad profesional porque va dentro de sus tareas, mismo que puede hacerse por escrito o verbalmente.

Por otro lado, en el Perú conforme el decreto ejecutivo N 728 la Ley de Productividad y Competitividad el contrato de trabajo es un conjunto de obligaciones que debe cumplir el empleador frente a la Superintendencia Nacional de Fiscalización laboral; en ese contexto los contratos ocasionales su duración máxima es de seis meses al año. 


\section{Metodología}

La investigación se desarrolló desde un paradigma crítico propositivo porque analizó el fundamento legal nacional como internacional referente al fenómeno jurídico que es la afectación del derecho al trabajo de los servidores públicos ocasionado por los contratos ocasionales en el Ecuador. Por tanto, ejecutó el enfoque cuali-cuantitativo, ya que estudió los conflictos sociales, por cuanto, examinó datos e información del número de servidores públicos que son contratados bajo la modalidad del contrato ocasional en el Ecuador.

Adicionalmente se utilizó la fuente primera como es escrito por la persona y la fuente secundaria porque facilita el proceso de consulta. Posteriormente, para la recolección de la información se aplicaron entrevistas semiestructuradas a tres profesionales del derecho como a dos servidores públicos de diferentes entidades públicas que conocen ampliamente la problemática de estudio, por el desempeño de sus laborales académicas, profesional y experiencia.

\section{Resultados}

El Estado ecuatoriano a través de las diversas instituciones que la conforman tienen la obligación legal como jurídica de proteger los derechos de cada trabajadora y trabajador, por ende, el organismo encargado es el Ministerio de Trabajo, el mismo que es una institución nacional con dependencia del poder ejecutivo, es decir, de la presidencia como tal, que tiene como misión servir en sus competencias, una de ellas es regular las remuneraciones del sector público; en cumplimiento de sus obligaciones para las cuales se crearon. De lo expuesto, se puede interpretar que estudia, resuelve y comprende cada una de las problemáticas que se entablan en el ámbito laboral y sobre todo hace conocer los derechos laborales de la ciudadanía con enfoque integrado de igualdad.

Los contratos ocasionales se dan por terminado unilateralmente por la entidad contratante debido al recorte de personal. De tal manera, este derecho se está viendo afectado en el Ecuador, por lo que debe estar regularizado por el Ministerio de Trabajo; mismo que dentro de sus objetivo estratégicos del 2015 al 2018 debe incrementar el trabajo digno en igualdad de oportunidad y trato, incrementar el cumplimiento de derechos y obligaciones de la ciudadanía laboral, acrecentar la eficiencia operacional como por último incrementar el uso eficiente del presupuesto ya sean físicos y tecnológicos como el desarrollo del talento humano, en ese contexto si damos cumplimiento a aquello, los contratos ocasionales se terminarían a nivel nacional, ya que mejorará la calidad gradual de estabilidad laboral, equitativa en el sector público donde con los resultados expuestos se observa el gran número de personas que no cuentan con un empleo en crecimiento, sino que más bien es por un tiempo corto y sin igualdad de oportunidades.

Para que no se vulnere el derecho al trabajo producto de los contratos ocasionales debe existir un control efectivo con el fin de concientizar a los trabajadores y empleadores sobre los derechos y obligaciones de este contrato, con el propósito de diseñar políticas y normativas que se centren en la realidad y fomente la igualdad de oportunidades. En ese contexto, los mecanismos de la Gestión institucional deben ser integrales y eficientes como la planeación, programación, uso, transparencia y evaluación en su modelo con estándares de calidad interna como externa.

En ese sentido, el organismo antes mencionado, se enfoca en tres áreas en la práctica una es bajar los índices de desempleo y subempleo; por cuanto, conforme la encuesta nacional de empleo, desempleo y subempleo (ENEMDU) determinó que, la tasa de desempleo a fecha marzo del 2019 es del 4,6\% a nivel urbano y rural en $2,2 \%$ y por otro lado el subempleo en el área urbana es del $18,5 \%$ y rural del $19,1 \%$, esto debido que en el Ecuador existe un índice alto de lo dicho, por lo que debe crearse nuevas modalidades de contrato y atacar la problemática de desempleo en los jóvenes profesionales. Tal es el caso que el Gobierno de turno sigue trabajando, pero hasta ahora hay pocos resultados, a su vez para cambiar este panorama debe haber nuevas inversiones y emprendimiento conforme una modalidad amigable y en fiel cumplimiento de los derechos de los trabajadores.

El centro de los contratos ocasionales es la estabilidad laboral, según Cabanellas (2003), la estabilidad del empleo, es considerado una subsistencia para el trabajador mientras cumpla lo establecido en la ley, otorgándole derechos: vacaciones, indemnizaciones por pre-aviso y despido, la conservación del empleo en caso de un accidente o enfermedad. Del concepto propuesto, se hace referencia que la palabra estabilidad es equilibrio, solidez, continuidad en su cargo con todos los beneficios otorgándole beneficios por la relación laboral.

Saavedra y Maruyama (2000), exterioriza que se entiende que la estabilidad laboral debe ser entendida como absoluta, por lo que fue reemplazada por la protección contra el despido arbitrario eliminándose la reposición del trabajador en el empleo. Es decir, la estabilidad laboral se ha constituido en diferentes legislaciones en la normativa constitucional con el fin que sea de directo y efectivo cumplimiento en beneficio de los trabajadores.
Por otro lado, el derecho del trabajo está regulado por un conjunto de normas y principios (Constitución del Ecuador, LOSEP, Código de Trabajo, Carta Andina, Convenio OIT, entre otras), por lo que legalizan las relaciones jurídicas que surgen del trabajo de prestación de servicios, dependencia y remunerado. Sin embargo, el servidor público es una persona humana que es indispensable en la administración pública, ya que el Estado a través de las diversas instituciones que la conforman contrata aquellas, con el fin de prestar servicios administrativos en beneficio de la colectividad. Es decir, el derecho al trabajo se ve configurado cuando el Estado se ve en la necesidad de contratar una persona la misma que realice una actividad en sociedad otorgándole derechos y obligaciones tanto a nivel nacional como internacional.

Cabe mencionar que, de estos, unos 30.000 deberán ir obligatoriamente a concursos porque la idea es regularizar su situación. Cabe decir, que con fecha 25 de junio, conforme el Ministerio de Trabajo se hace hincapié que no se puede contratar de esa forma, salvo excepción debidamente justificada. Por tanto, ninguna entidad pública podrá superar el plazo de doce meses máximo de los contratos de servicios ocasionales (Ledesma, 2018).

Cabe señalar que conforme a Ledesma (2019): hasta el 16 de enero del 2019 las instituciones públicas tienen la obligación legal de regularizar las nuevas contrataciones ocasionales ante el Ministerio del Trabajo, por medio de un control endurecido para el cumplimiento de las reglas vigentes. Según el acuerdo Ministerial MDT 2019 001, se aclara en tal sentido que sí se podrán realizar nuevos contratos ocasionales, siempre y cuando la entidad que lo posea cree una asignación presupuestaria para el efecto, cumpliendo los requisitos preestablecidos, con el fin de cubrir una necesidad no permanente. De todas maneras, quienes tienen más de cuatro años sí tendrán una prórroga de su contrato hasta la creación de un nuevo puesto permanente.

La normativa hizo reformas a esa figura de contratación, que a criterio del ministro fue usada "de manera totalmente irregular y en desmedro de los empleados, por lo que existen funcionarios que llevan trabajando para el Estado por más de 6 años, cuando este tipo de contratos duran un año y son para el desarrollo de una actividad especial que no será permanente; Eso es precarización. Además, se debe tomar en cuenta que los servidores eran perjudicados porque no se les brindaba estabilidad laboral, ya que en cualquier momento podían ser cesados. Por ejemplo, en el Consejo Nacional Electoral (CNE) hay cerca de 500 empleados con contratos ocasionales que en algunos casos superan los 4 años (Ledesma, 2018).

\section{Conclusiones}

Para frenar la precariedad de los contratos ocasionales a nivel nacional como regional lo pertinente es la aplicación de la norma técnica de subsistema de selección de personal en concordancia con los artículos 11, 61 y 228 de la Constitución de la República del Ecuador, tal es el caso, que en la práctica se requiere la existencia de la necesidad institucional, posteriormente se elabora un informe técnico que es remitido a la máxima autoridad, con el fin que se aprueba una planificación para llamar al concurso de méritos y oposición, a su vez se añadirà el perfil al puesto y sus respectivas actividades ha desarrollarse, como la disponibilidad presupuestaria.

Los concursos de méritos y oposición deben contar con profesionales altamente calificados para su ejecución y cumplimiento conforme lo establece la sección II administración pública del art. 228 de la Constitución de la República del Ecuador. Por ejemplo, empieza con la preparación del proceso, convocatoria, evaluación y selección, partida presupuestaria, cumplir lo que establece la LOSEP y su Reglamento, debe contar con 3 aspirantes, caso contrario se declara desierto, posteriormente en caso de duda se resuelve ante el tribunal de méritos y oposición y por ultimo se observarán los principios y disposiciones señaladas en la norma sustitutiva de la norma del subsistema de reclutamiento y selección de personal.

Debe aplicarse este procedimiento por parte de las entidades nominadoras, por lo que, en el año 2017 y 2018 alrededor de 187. 635 (ciento ochenta y siete mil con seis cientos treinta y cinco) servidores públicos son contratados bajo la modalidad de contratos ocasionales. Por ende, la administración pública conformada por la función Ejecutiva, Función Electoral, Función Judicial, Función Legislativa, Función de Transparencia y Control Social, Instituciones creadas por la Constitución y la ley y por último los Gobiernos Autónomos Descentralizados, por lo que no garantiza el cumplimiento del derecho al trabajo, vida digna, buen vivir, estabilidad económica.

Este estudio se ha enfocado en la contratación ocasional, mismo después de los doce meses son separados de su lugar de trabajo los servidores, por lo que, muchos de estos no son renovados puesto que no existe partida presupuestaria para volverlos a contratar, es decir, esto repercute que no se dé cumplimiento al plan nacional del buen vivir o conocido como Sumak Kawsay, por lo que si no cuentan con estabilidad laboral no podrán sustentar a sí mismo como a su familia. El Estado debe contar con acciones eficientes en favor del derecho al trabajo, 
para con ello responder las necesidades de la sociedad, con el propósito de promover o impulsar los concursos de méritos y oposición y a su vez debe codificarse la normativa en concordancia con el plan nacional del buen vivir a través de sus doce objetivos como por ejemplo uno de ellos es el principal agente de acción colectiva, en busca de recuperar el país para la ciudadanía y también fomentar la acción colectiva de la propia sociedad. Siendo parte del respeto a la autonomía de las organizaciones sociales y se reconoce el papel del Gobierno para promover la participación social y ciudadana con el fin de mantener a sus familias.

El Ecuador a partir de la formación o creación de la Constitución del 2008, se interpreta que todos los derechos están reconocidos y serán respetados, garantizados y de estricto cumplimiento y sobre todo obligatorios en beneficio del ciudadano o ciudadana, uno de ellos es el derecho al trabajo y el buen vivir a través de un trabajo digno y sujeto a una remuneración; misma que servirá para desarrollar su vida en sociedad.

Uno de los problemas determinados consiste en que las entidades públicas no proveen los principios constitucionales establecidos en la Constitución del Ecuador, lo cual vulnera el principio de igualdad, continuidad laboral, irrenunciabilidad e intangibilidad y no discriminación, por cuanto, todos los servidores públicos deben ser contratados de la misma forma que todos, sin embargo, muchos de ellos se ven en la necesidad y en la obligación de suscribirse debido a la falta de trabajo en el Ecuador; privándose de ciertos derechos como uno ellos es permiso, licencias para estudio, etc.

Por medio de las entrevistas semiestructuradas aplicadas a abogados especialistas en materia laboral, se determinó que existe vulneración a lo establecido en el art. 11 de la Constitución del Ecuador referente que la ley sancionará toda forma de discriminación, según lo expuesto se discrimina a ciertos servidores públicos por no pertenecer a la carrera administrativa, porque bajo la modalidad de ocasional no ingresan a la administración pública por la falta de ejecución de los concursos de méritos y oposición.

Además, los contratos ocasionales no permiten la superación académica, por lo que se ha probado que afecta al normal desempeño de los funcionarios, por tanto, debe existir permisos, licencias y beneficios académicos con el fin de brindar un servicio de calidad a la sociedad, por al menos 2 horas diarias. Por último, se debe impulsar la estabilidad del servidor público por medio de planes de capacitación, reformas legales encaminadas a garantizar el cumplimiento de los derechos de las personas que conforman la administración pública.

Respecto a la legislación comparada los contratos ocasionales en Colombia son utilizados de manera legal y válida, puesto que se prevé el derecho al trabajo y garantías como el buen sueldo, lo cual no viola las disposiciones legales debido al tiempo y a su utilización correcta. Mientras tanto, en el Ecuador se utiliza abusivamente por un tiempo de un año, mismos que no proporcionan una estabilidad laboral a los trabajadores y dejan sin el sustento diario a las familias, por cuanto, se debe optar por la denominación de un cierto tiempo no mayor inclusive a cuatro (4) meses como se aplica en los países de Colombia y Perú.

Otro dato relevante, conforme la Segunda Sala de lo contencioso Administrativo, 2008; la norma jurídica no se armoniza con el servidor público, por lo que fue codificada por más de ocho ocasionales debido a la no estabilidad laboral siendo un derecho constitucional y legal de mayor relevancia. En ese contexto, la acción de protección N 189-10 reconoce que se vulnera el derecho de igualdad material y formal, derecho a la libertad de contratación de los ciudadanos u ciudadanas.

Finalmente, los contratos ocasionales, mismos que cumplen los requisitos de un contrato de trabajo conforme el Código de trabajo, el mismo que prevé un salario, dependencia, a su vez en su parte primera están los antecedentes, segunda objeto del contrato según el art. 17 de cuerpo legal antes mencionado, tercera la remuneración, cuarta duración, quinta lugar de trabajo, sexta obligaciones del trabajador y el empleador, estos se sujetan estrictamente a lo dispuesto en el Código de Trabajo en su Capítulo IV, séptima la legislación aplicable, octava la jurisdicción y competencia, estas se someterán a los jueces competentes del lugar en que este contrato ha sido celebrado y por último la suscripción en la que las partes se ratifican en todas y cada una de las cláusulas precedentes y para constancia firman el contrato original al tenor del acuerdo, es decir, conforme lo expuesto al existir un control directo de la persona contratada por esta modalidad debe otorgarse todos los beneficios de ley.

\section{Referencias}

Abad, E. (2016). El derecho a la estabilidad de los servidores públicos con contrato ocasional. Recuperado de http://repositorio.pucesa.edu.ec/bitstream/123456789/1823/1/76326.pdf

Balbín, A. (2015). El concepto de derecho del trabajo. Revista Anales de la

Facultad de Ciencias Jurídicas y Sociales U.N.L.P.01, (364).

Cabanellas, G. (2003). Diccionario Jurídico Elemental. Editorial Heliasta.

Carta Andina, P. (02 de 07 de 2002). Carta Andina para la Promoción y Pro- tección de los Derechos Humanos. Guayaquil, Ecuador.

Ecuador. Leyes y Decretos. (s.f). Código del Trabajo. 1e. Quito: Ecuador. Ecuador. Leyes y Decretos. (s.f). Constitución de la República del Ecuador. 1e. Quito: Ecuador.

Ecuador. Leyes y Decretos. (s.f). Ley Orgánica del Servicio Público. 1e. Quito: Ecuador

García, P. (2012). Derecho Laboral. Recuperado de http://fcasua.contad. unam.mx/apuntes/interiores/docs/2012/administracion/2/derecho_laboral.pdf

Gavilánez, A. (2018). El contrato de prestación de servicios ocasionales y el principio de estabilidad laboral en el sector público. Recuperado de http://repositorio.uta.edu.ec/bitstream/123456789/27466/1/FJCS-DE-1959.pdf

Maldonado, J. (2017). Análisis de los contratos de trabajo y el respeto al derecho del trabajador enmarcados en la constitución de la República. Recuperado de http://repositorio.utmachala.edu.ec/bitstream/48000/11186/1/E7407_ MALDONADO\%20JORDAN\%20JIMMY\%20JOSE.pdf

Montserrat, J. (2014). Estabilidad laboral y flexiseguridad. Observatorio Laboral Revista Venezolana. Vol. 7, número. 14

Muñoz, M. (2014). Los Regímenes Jurídicos que regulan las Relaciones Laborales y de Servicios entre los Servidores Públicos y el Estado ecuatoriano, a par tir de la promulgación de la Constitución de 2008. Recuperado de http://www. dspace.uce.edu.ec/bitstream/25000/3938/1/T-UCE-0013-Ab-225.pdf

Ostau de Lafont, F. (2009). Tratado de Derecho del Trabajo. Bogotá: Ibañez.

Parra, W. (2005) Empleo Público, Gerencia Pública y Gerencia Administrativa. Bogotä: Ediciones Jurídicas Gustavo Ibañez.

Peralta, M. Santofimio, A. \& Segura, V. (2007) El Compromiso Laboral: Discursos en la organización. Desde el Caribe.

Saavedra, J. \& Maruyama, E. (2000). Estabilidad laboral e indemnización: efectos de los costos de despido sobre el funcionamiento del mercado laboral peruano. Recuperado de http://biblioteca.clacso.edu.ar/Peru/ grade/20100708115641/ddt28.pdf

Salazar, M. (2015). Inexistencia de protección e iqualdad de derechos de los servidores públicos con contratos de servicios ocasionales en relación a los servidores públicos con nombramiento, en la actual Ley Orgánica del Servicio Público. Recuperado http://www.dspace.uce.edu.ec/bitstream/25000/4969/1/TUCE-0013-Ab-306.pdf

Valencia, $R$. (2016). Los contratos de servicios ocasionales y la violación a los derechos laborales en las instituciones públicas del Ecuador. Recuperado de http://dspace.utpl.edu.ec/bitstream/123456789/15516/1/Valencia_Cuvina_Ri cardo Israel.pdf 